\title{
Frustration effect as a function of training magnitude in a within-S design'
}

JAMES H. MCHOSE, PHILIP A. MEYER, ${ }^{2}$ and FREDERICK R. MAXWELL, JR., Southerm Illinois University, Carbondale. Ill. 62901

Incidental findings from two studies in which rats were differentially conditioned in the first alley (A1) of the L-shaped double alley apparatus and subsequently administered nonreward $(N)$ test trials in both the positive $(S+)$ and negative $(S-)$ discriminandum are reported. The speeds with which $S$ s ran the second alley following an $N$ trial were, for two groups, independent of whether $N$ occurred in $S+$ or $S-$; for a third group speeds were faster following $N$ in $S$ - than following $N$ in $S+$.

Several studies have shown that nonreinforcement of a previously reinforced response augments subsequent responding. Thus in the double-alley apparatus rats regularly reinforced in the first goal box (G1) run faster in the second alley (A2) following omission of G1 reward than do Ss never reinforced in Gl (Barrett et al, 1965; Wagner, 1959). The facilitative effect of reward omission has been attributed to the occurrence of frustration $\left(R_{F}\right)$ on reward omission trials which augments the general drive level of the organism. Within the model developed by Amsel (1958) the intensity of $R_{F}$ and thus the amount of drive increment resulting from reward omission is assumed to be a positive function of the organism's reward expectancy level $\left(r_{g}\right)$. Since $r_{g}$ is assumed to be directly related to the amount of reinforcement received in $G 1$ on rewarded (R) trials, A2 speeds on nonrewarded $(\mathrm{N})$ trials should vary directly with the magnitude of reward received in G1 on $R$ trials.

Two basic experimental paradigms have been used in an attempt to ascertain the relationship between $\mathrm{R}$-trial magnitude in $G 1$ and $A 2$ speeds following $N$ trials in $G 1$. In the between-S design, different groups of Ss have been trained on differing magnitudes of $\mathrm{Gl}$ reward prior to the omission of reward in G1 on test trials. Studies employing this paradigm. have reported either no difference in A2 speeds on $\mathrm{N}$ trials as a function of R-trial magnitude (McHose \& Ludvigson, 1965) or an inverse relationship between $\mathrm{R}$-trial magnitude and $\mathrm{N}$-trial speeds (Barrett et al, 1965). In the within-S design one group of Ss receives differential conditioning training in the first alley (A1) of the double alley apparatus, reward magnitude varying with, for example, brightness of $A 1$. Thus each $S$ receives a high magnitude of $G 1$ reward in one discriminandum (S+) and a low reward magnitude in $\mathrm{Gl}$ in the other discriminandum $(\mathrm{S}-$ ). After discrimination training, Ss are administered $N$ test trials in both $S+$ and $S-$. Using the within-S paradigm, Peckham \& Amsel (1967) found A2 speeds following $\mathrm{N}$ in $\mathrm{S}+$ to be faster than those following $\mathrm{N}$ in $\mathrm{S}$-. The between- and the within-S designs, then, have yielded conceptually discrepant results with respect to the expectancy-frustration dependency postulated by Amsel (1958). The present paper reports portions of two experiments employing the within-S design, the results of which suggest that the apparent discrepancy must reflect differences in previous studies other than those inherent in the between-as opposed to the within-S design.

\section{METHOD}

In Experiment 1, 24 male albino rats, 100-120 days old at the beginning of the experiment, received 80 acquisition and 32 postshift trials in a modified L-shaped double alley apparatus. The first section of the double alley apparatus was comprised of two parallel, moveable alley (A1) and goal (G1) sections, one painted flat-black, the other flat-white. Moving this section of the apparatus permitted alignment of either the black or white alley and goal sections with a mid-grey start box (S1). The lengths of $S 1, A 1$, and $G 1$ were 10,32 , and 10 in., respectively. A mid-grey orienting chamber (S2), 11 in. long, adjacent to $G l$ preceded the mid-grey second runway which consisted of a 36 -in. long alley (A2) and 10-in. goal box (G2). The interior width of the apparatus was 4 in. throughout, and the height $3 \frac{1 / 2}{\mathrm{in}}$. The apparatus had the following doors: solenoid-operated, opaque side exit doors separating $S 1$ from $A 1, G 1$ from $S 2$, and $S 2$ from $A 2$, and guillotine transluscent doors separating $A 1$ from $G 1$ and $A 2$ from G2. Opening the first start door initiated a clock which stopped when $S$ interrupted a photocell beam located 12 in. into $\mathrm{Al}$, providing an $\mathrm{Al}$ start measure. Clock and photo electric circuitry also provided traversal times over the first 6 in. and next 12 in. of $A 2$.

All Ss were placed on a 23-h food deprivation cycle 12 days prior to the first experimental day (Day 13). On Days 11 and 12 , approximately $1 \mathrm{~g}$ of $45-\mathrm{mg}$ Noyes pellets, identical with the subsequent reinforcement pellet, was incorporated in to Ss' daily diet, and Ss were allowed to explore the S1 and Al portions of the apparatus for $5 \mathrm{~min}$.

Two groups (Groups 8-1 and 4-1) were randomly constituted and designated according to the number of pellets received in the first alley on each $\mathrm{S}+$, e.g., black $\mathrm{Al}-\mathrm{Gl}$, and each $S_{-}$, e.g., white $A 1-G 1$, acquisition trial. During the postshift period, group reinforcement conditions were unchanged except that both groups received one trial per day on which reward was omitted from Gl, either on an St or an $\mathrm{S}-$ trial. Within each block of 16 trials, each $\mathrm{S}$ received four nonrewarded $(\mathrm{N})$ trials in $\mathrm{St}$ and four $\mathrm{N}$ trials in $\mathrm{S}-$, with daily trial position of $N$ trial counterbalanced. The brightness of $\mathrm{S}+(\mathrm{S}-)$ was counterbalanced between Ss within each group. Ss received four trials per day with the brightness of A1 varied according to the following repeating cycle: BWBW. WWBB, WBBW, BBWW, WBWB, BWWB. Trials were administered to $S s$ in squads of eight, with the order of trial administration randomized from day to day. All Ss always received two pellets of immediate reward in $\mathrm{G} 2$.

On any trial, the first start door opened after $S$ had maintained an orientation toward the door for $3 \mathrm{sec}$. The door separating $\mathrm{G} 1$ from $\mathrm{S} 2$ was opened as soon as $\mathrm{S}$ withdrew from the (empty) goal cup in Gl; the second start door opened after a $3-\mathrm{sec}$ orientation by $\mathrm{S}$.

The apparatus, deprivation, habituation, and general trial administration procedures of Experiment 2 were identical to those of Experiment 1. Only one group of $10 \mathrm{Ss}$, Group 9-4, labeled analogous to the group designation of Experiment 1, received $N$ trials during the postshift period. Group 9-4 received 160 acquisition trials followed by 48 postshift trials. This experiment also contained a 9-1 group, the data for which are not relevant to the present paper.

In both experiments, starting times in $\mathrm{A} 1$ were reciprocated and start and run traversal times in A2 on each trial were combined and reciprocated, yielding an Al starting speed and an A2 "total speed" measure, respectively.

\section{RESULTS}

Group mean A2 total speeds on $\mathrm{N}$ trials for the various conditions of Experiment 1 are plotted as a function of blocks of 16 trials in Fig. 1. Within each block of trials the mean speeds for Groups 8-1 and 4-1 on the two $\mathrm{N}$ trials occurring in 


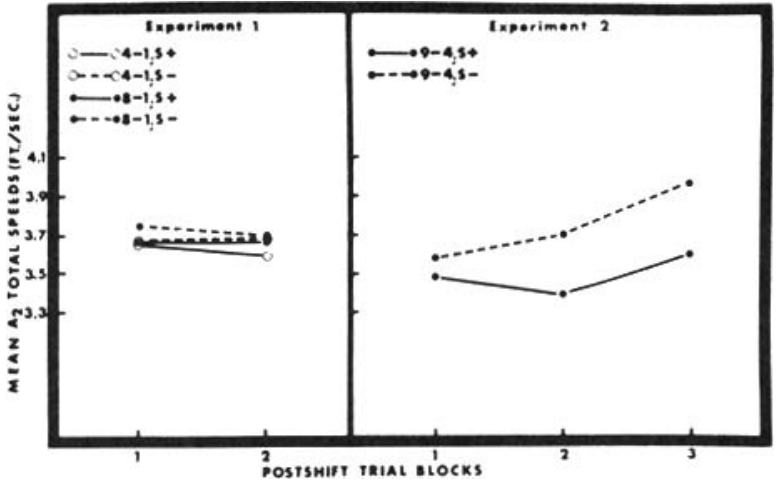

Fig. 1. Men A2 speeds on nonreinforced trials as a function of triak for the various conditions of Experiments 1 and 2.

$\mathrm{S}+$ are plotted separately from the mean speeds on the two $\mathrm{N}$ trials occurring in $\mathrm{S}$-. As may be seen, neither group displayed appreciably different $A 2$ speeds following an $\mathrm{N}$ trial in $\mathrm{S}+$ as compared with $\mathrm{S}-$. Moreover, the A2 speeds of Group 8-1 following an $\mathrm{N}$ in $\mathrm{S}+$ do not appear to differ from those of Group 4-1 following an $\mathrm{N}$ on the four-pellet $\mathrm{S}+$. A four-factor variance analysis containing two within (Blocks 1 vs 2 and $\mathrm{S}+$ vs $S-$ ) and two between (Groups $8-1$ vs $4-1$ and Black vs White $\mathrm{S}+$ ) sources of variance was performed on the $\mathrm{N}$-trial data presented in Fig. 1. This analysis yielded $F$ ratios less than unity for the Groups and the $S+v s ~ S-$ factors, as well as their interaction. Similarly, all interactions between these sources of variance and the trial blocks factor had associated $F$ ratios less than unity.

In order to ascertain whether the differential reinforcement in $\mathrm{GI}$ produced faster $\mathrm{A} 1$ speeds in $\mathrm{S}+$ as compared with $\mathrm{S}$-, the postshift Al starting speeds were subjected to a four-factor variance analysis identical to the previous $\mathrm{N}$-trial analysis. This analysis yielded a significant $(p<.01) S+$ vs $S-$ effect $(F=54.72, \mathrm{df}=1 / 20)$, Ss starting significantly faster in $S+$ as compared with $\mathrm{S}-$.

The mean A2 total speeds on $N$ trials for Groups $9-4$ of Experiment 2 are also plotted as a function of blocks of 16 trials in Fig. 1. As may be seen, speeds following $N$ in the four-pellet ( $\mathrm{S}_{-}$) $\mathrm{G} 1$ were considerably above those following $\mathrm{N}$ in the nine-pellet (S+) G1. Variance analysis of these data over the three postshift trial blocks yielded a significant $(p<.01)$ $S+$ vs $S$ - effect $(F=11.54, \mathrm{df}=1 / 10)$. The Blocks effect was also significant $(F=17.35, \mathrm{df}=2 / 20)$, but the Blocks by $S+$ vs $\mathrm{S}$ - interaction did not approach statistical significance.

Finally, variance analysis of Al starting speeds during the postshift period yielded no significant difference between the speeds of Group 9-4 in the S+ as compared with the S- first alley.

\section{DISCUSSION}

In Experiment 1, A2 speeds following nonreward $(N)$ in $G 1$ were unrelated to the amount of reward received in $\mathrm{G} 1$ on rewarded trials. Thus both Groups $8-1$ and 4-1 displayed speeds following $\mathrm{N}$ in $\mathrm{S}+$ which approximated their speeds following $N$ in $S-$. For Group $9-4$ (Experiment 2), however, $N$ in $\mathrm{S}$ - produced faster speeds than did $\mathrm{N}$ in $\mathrm{S}+$. As previously outlined (Peckham \& Amsel, 1967), it may be deduced from the basic assumptions of Amsel's (1958) frustration model that, in the within-S design, omission of $\mathrm{Gl}$ reward will elevate A2 speeds in proportion to the reward magnitude regularly received in that goal box. Neither the results of Experiment 1 nor those of Experiment 2 are supportive of the frustration model; indeed, those of Experiment 2 are directly opposite to the predictions derivable from the model.

Several inconsistencies exist within the literature relevant to the expectancy-frustration dependency postulated in Amsel's frustration model. In the present data, the results of Experiment 2 differ from those of Experiment 1. Secondly, both experiments produced results inconsistent with data obtained in two separate experiments reported by Peckham \& Amsel (1967). The observation that Groups 8-1 and 4-1 (Experiment 1) formed a discrimination in $\mathrm{Al}$, i.e., ran faster in $\mathrm{S}+$ than in $\mathrm{S}$-, while Group $9-4$ (Experiment 2) did not, may be related to the disparate $A 2$ results between Experiments 1 and 2. In this connection, Peckham and Amsel's data also suggest that a well-formed as compared with a nonsignificant Al discrimination, as measured by speeds in $A 1$, elevates $A 2$ speeds on $S+N$ trials relative to those on $S-N$ trials. While the different experimental results of Experiments 1 and 2 as well as the differences between the first and second experiments reported by Peckham and Amsel may be related to the extent of discrimination in $\mathrm{Al}$, the disparity between the present results and those of Peckham and Amsel would appear to hinge upon some other subtle differences in experimental procedure.

Finally, it should be noted that the present data suggesting either no relationship or an inverse relationship between reward magnitude received on $\mathrm{R}$ trials and speeds on $\mathrm{N}$ trials are consistent with the type of data typically, albeit not universally (cf. Krippner et al, 1967) obtained in the between-S design analog (Barrett et al, 1965; McHose \& Ludvigson, 1965). Unfortunately, the present results serve to further confuse the question of how, in frustration terms, frustration varies with expectancy. They strongly suggest, however, that the differences between results obtained in previous between-S as opposed to within-S designs are not attributable to the greater "sensitivity" of the within-S design or any other systematic difference between the within-S and between-S designs.

\section{REFERENCES}

AMSEL, A. The role of frustrative non-reward in non-continuous reward situations. Psychological Bulletin, 1958, 55, 102-119.

BARRETT, R. J., PEYSER, C. S., \& McHOSE, J. H. Effects of complete and incomplete reward reduction on a subsequent response. Psy chonomic Science, 1965, 3, 277-278.

KRIPPNER, R. A., ENDSLEY, R. C., \& TACKER, R. S. Magnitude of $G_{1}$ reward and the frustration effect in a between subjects design. Psychonomic Science, 1967, 9, 385-36.

McHOSE, J. H., \& LUDVIGSON, H. W. Role of reward magnitude and incomplete reduction of reward magnitude in the frustration effect. Journal of Experimental Psy chology, 1965, 70, 490-495.

PECKHAM, R. H., \& AMSEL, A. Within-subjects demonstration of a relationship between frustration and magnitude of reward in a differential magnitude of reward discrimination. Journal of Experimental Psychology, 1967, 73, 187-195.

WAGNER, A. R. The role of reinforcement and non-reinforcement in an "apparent frustration effect." Journal of Experimental Psychology, $1959,57,130-136$.

\section{NOTES}

1. Supported by Research Grant MH-10340 and Training Grant MH 07817 from the United States Public Health Service.

2. Now at the University of South Dakota. 